\title{
Energy barriers and driving forces in tRNA translocation through the ribosome
}

\author{
Lars V Bock ${ }^{1,8}$, Christian Blau ${ }^{1,8}$, Gunnar F Schröder ${ }^{2,3}$, Iakov I Davydov ${ }^{4}$, Niels Fischer ${ }^{5}$, Holger Stark ${ }^{5,6}$, \\ Marina V Rodnina ${ }^{7}$, Andrea C Vaiana ${ }^{1} \&$ Helmut Grubmüller ${ }^{1}$
}

\begin{abstract}
During protein synthesis, tRNAs move from the ribosome's aminoacyl to peptidyl to exit sites. Here we investigate conformational motions during spontaneous translocation, using molecular dynamics simulations of 13 intermediate-translocation-state models obtained by combining Escherichia coli ribosome crystal structures with cryo-EM data. Resolving fast transitions between states, we find that tRNA motions govern the transition rates within the pre- and post-translocation states. Intersubunit rotations and L1-stalk motion exhibit fast intrinsic submicrosecond dynamics. The L1 stalk drives the IRNA from the peptidyl site and links intersubunit rotation to translocation. Displacement of tRNAs is controlled by 'sliding' and 'stepping' mechanisms involving conserved L16, L5 and L1 residues, thus ensuring binding to the ribosome despite large-scale tRNA movement. Our results complement structural data with a time axis, intrinsic transition rates and molecular forces, revealing correlated functional motions inaccessible by other means.
\end{abstract}

Ribosomes are molecular machines that synthesize proteins from aminoacyl tRNAs, using mRNA as template. After formation of a peptide bond, the two tRNAs bound to the aminoacyl (A) and peptidyl (P) sites on the small (30S) and large (50S) ribosomal subunits translocate by more than $7 \mathrm{~nm}$ to the $\mathrm{P}$ and exit (E) sites, respectively, while the next mRNA codon moves into the A site (Fig. 1a). During translocation, tRNAs move on the 50S subunit into the hybrid A/P and $\mathrm{P} / \mathrm{E}$ positions ${ }^{1}$ with a concomitant rotation of the $30 \mathrm{~S}$ subunit relative to the $50 \mathrm{~S}$ subunit ${ }^{2-4}$. The rate-limiting step of translocation is the displacement of the codon-anticodon complexes on the $30 \mathrm{~S}$ subunit; this, followed by the reversal of the subunit rotation, yields the post-translocation complex. Translocation is promoted by elongation factor G (EF-G) and is driven by GTP hydrolysis. In the absence of the factor, spontaneous, thermally driven tRNA translocation can occur $^{5-8}$, and this seems to involve the same intersubunit interactions that occur in the presence of EF-G $\mathrm{G}^{9}$. Spontaneous translocation is an equilibrium process, in which the tRNAs make rapid, spontaneous excursions in both forward and backward directions ${ }^{5,6,10}$. Preferential directionality is determined by the affinities of the tRNAs for their respective binding sites ${ }^{5,6}$. The process of translocation entails fluctuations of tRNAs ${ }^{4,11-14}$ and of the components of the 50S subunit such as the L1 stalk ${ }^{3,15-17}$. A recent cryo-EM work revealed a large number of different conformational states for spontaneous, thermally driven tRNA movement through the ribosome ${ }^{10}$. However, precisely how the thermal fluctuations of tRNAs and of parts of the ribosome cooperatively drive the tRNA movement is unclear. It is also unclear whether and how synchronous movements-such as those involving intersubunit rotations, the $\mathrm{L} 1$ stalk and $\mathrm{tRNA}^{\mathrm{fMet}}$ - are coupled to one another. Furthermore, it is unknown how efficient tRNA handover from one binding site to another is achieved, despite the considerable structural changes along the translocation path. To address these questions, we combined data from X-ray crystallography and singleparticle cryo-EM with molecular dynamics (MD) simulations.

\section{RESULTS}

Structural models of translocation intermediates

We refined crystal structures of E. coli ribosomes ${ }^{18}$ against 13 selected cryo-EM density maps ${ }^{10}$ of ribosomes assembled in the posttranslocation state, with P-site fMet-Val-tRNA ${ }^{\mathrm{Val}}(\mathrm{P} / \mathrm{P}$ state) and deacylated $\mathrm{tRNA}^{\mathrm{fMet}}$ in the $\mathrm{E}$ site, and the tRNAs spontaneously moved into their A/A and P/P states, respectively ${ }^{5}$ (Supplementary Fig. 1, Supplementary Video 1 and Supplementary Table 1, models and refinement described in Supplementary Note 1). Several flexible structure-refinement methods have been developed ${ }^{19-23}$. In a recent independent benchmark study ${ }^{24}$ comparing four methods ${ }^{20-23}$, the DireX refinement method used here was shown to produce on average the most accurate results ${ }^{23}$. All four methods were shown to yield highquality atomic models from a combination of high-resolution crystal structures and low-resolution data. Indeed, an independent MDbased refinement of the pretranslocation (pre) state prelb cryo-EM

\footnotetext{
1Department of Theoretical and Computational Biophysics, Max Planck Institute for Biophysical Chemistry, Göttingen, Germany. ${ }^{2}$ Computational Structural Biology Group, Institute of Complex Systems (ICS-6), Forschungszentrum Jülich, Jülich, Germany. ${ }^{3}$ Department of Physics, Heinrich-Heine Universität, Düsseldorf, Germany. ${ }^{4}$ Scientific Research Center Bioclinicum, Moscow, Russia. 53D Electron Cryomicroscopy Group, Max Planck Institute for Biophysical Chemistry, Göttingen, Germany. ${ }^{6}$ Department of Molecular Electron Cryomicroscopy, Institute of Microbiology and Genetics, Georg-August Universität Göttingen, Göttingen, Germany. ${ }^{7}$ Department of Physical Biochemistry, Max Planck Institute for Biophysical Chemistry, Göttingen, Germany. ${ }^{8}$ These authors contributed equally to this work. Correspondence should be addressed to G.F.S. (gu.schroeder@fz-juelich.de), A.C.V. (avaiana@gwdg.de) or H.G. (hgrubmu@gwdg.de).
} 
Figure 1 Structural transitions and interactions along the translocation pathway. (a) Left, schematic of tRNA $A^{\text {Val }}$, tRNA ${ }^{\text {fMet }}$ and L1-stalk motions (indicated by arrows). Right, schematic of $30 \mathrm{~S}$ head and body rotations. (b) Backbone r.m.s. deviation (r.m.s.d.) between the MD structural models of 13 intermediate states of translocation (substates are denoted by a and $b$ ) and two crystal structures ${ }^{26}$ (solid, whole ribosome; dashed, tRNA-binding region). (c) Motions of tRNAs and L1 stalk quantified by reaction coordinates (R. c.) for the 13 states. Arrows denote directionality of motions as in a; black bars indicate the fluctuation range covered by each simulation; colored bands denote interaction enthalpies. (d) Estimated intrinsic transition rates (denoted by line thickness) between intermediate states of translocation (circles) for motions identified in a. (e) Changes in the angles of the 30 s head tilting, swiveling and $30 \mathrm{~S}$ body rotation. Black bars indicate the fluctuation range covered by each simulation. (f) Molecular driving forces between L1 stalk and tRNA. Two structures representing the range of distances from the fully closed (top left) to open (top right) conformations. Distance dependence of the potential of mean force (PMF, black line, with errors in gray) and interaction enthalpy (circles, colored according to state) between tRNA ${ }^{\text {fMet }}$ and L1 stalk. A mechanical analog of the repulsive (sketched gray line, red region) and attractive (green region) regimes is shown below.

map by one of the alternative methods ${ }^{25}$ resulted in a structure very similar to that obtained with DireX, with an r.m.s. deviation of $3.2 \AA$ (Supplementary Notes 2 and 3 ).

\section{Validation of the models}

We first assessed the quality of our structural models (Fig. 1b). The models of the prela state closely resemble the crystal structure of the ribosome trapped in the classical state ${ }^{26}$, with an r.m.s. deviation of $4.9 \AA$ for the whole ribosome. The structural changes during translocation between the classical state ${ }^{26}$ and the other pretranslocation states are captured by r.m.s. deviations of up to $11 \AA$. After the refinement was completed, the structure of one latepretranslocation state was reported ${ }^{26}$, with the P-site tRNA in the hybrid $\mathrm{P} / \mathrm{E}$ position and the ribosomal subunits rotated to a high degree; this offered the possibility of testing the quality of one of our structure predictions. Comparison of the pre 4 state with this crystal structure gave an r.m.s. deviation of $4.7 \AA$ for the whole ribosome complex and $3.5 \AA$ for the tRNA-binding region. Apparently, our refinement procedure yielded an accuracy of the pre 4 model similar to that of the classical pre 1 model directly derived from an $\mathrm{X}$-ray structure in the classical state. All other states showed larger deviations, as expected for structurally distinct intermediates. The positions of the tRNAs were accurately predicted, particularly in the functionally relevant anticodon and CCA-end regions (Supplementary Fig. 2a). These independent quality checks also suggest that the models of the other pre- and post-translocation states, for which no crystal structures exist, are similarly accurate. The agreement of the models' local stereochemical parameters with those of available crystal structures (Supplementary Table 2) confirms their similar stereochemical quality.
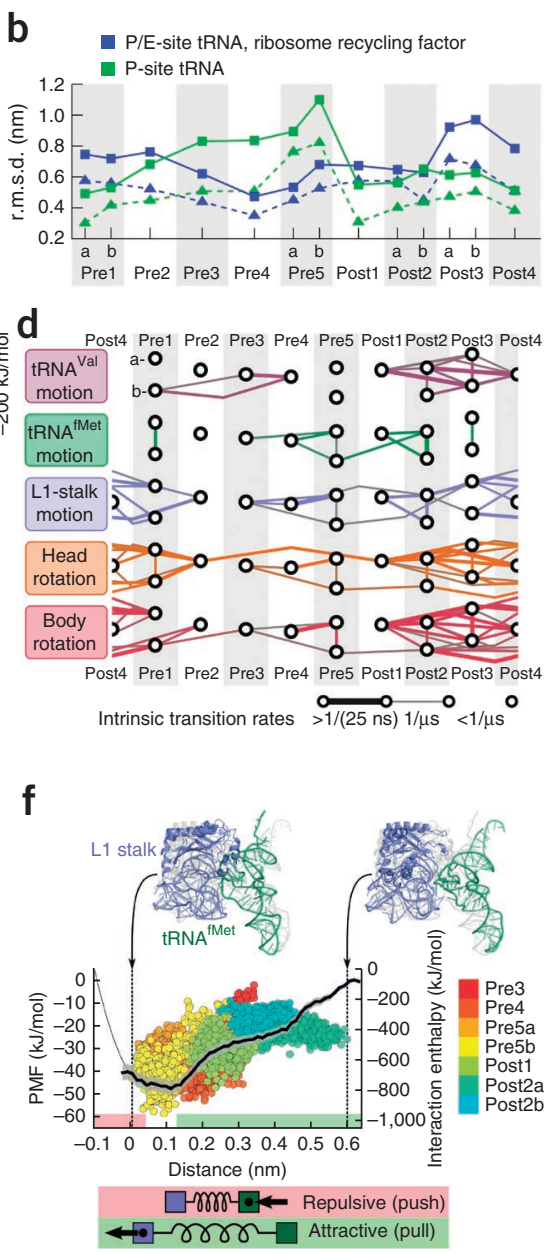

\section{Large-scale conformational motions of the ribosome}

The 13 structural models, grouped into nine major pre- and posttranslocation (post) states on the basis of tRNA positions, revealed details of tRNA movement as well as large collective motions of the whole ribosome on slow timescales, of seconds to minutes, accessible to the cryo-EM experiments. Structural transitions and interactions along the translocation pathway are shown in Figure 1. Six selected motions are indicated (Fig. 1a) and characterized (Fig. 1c,e). The movement of tRNA ${ }^{\mathrm{Val}}$ between the $\mathrm{A}$ and $\mathrm{P}$ sites and of tRNA ${ }^{\text {fMet }}$ between the $\mathrm{P}$ and $\mathrm{E}$ sites as well as the concomitant movement of the L1 stalk (comprising L1 protein bound to nucleotides 2084-2206 of 23S rRNA) are quantified (Fig. 1c).

Understanding of how the ribosome controls tRNA translocation requires (i) resolution of its fast conformational motions, (ii) determination of the rates of these motions and (iii) uncovering of the underlying molecular driving forces. To this end, we carried out all-atom explicit-solvent MD simulations of the entire ribosome for the 13 preand post-translocational models ( Supplementary Video 2, MD setup and simulations described in Supplementary Note 1). The system comprised $\sim 2.2$ million atoms, and the simulations spanned a total of $>1.8 \mu$ s. The r.m.s. deviation during the equilibration simulations of each of the 13 structural models served as a third independent control (Supplementary Fig. 2b). Large r.m.s. deviations have been shown to point to inaccurate structures ${ }^{27}$. For all simulations, the r.m.s. deviations from the respective starting structure remained small and comparable to the deviations in the simulation started from the crystal structure.

We first analyzed the global swiveling and tilting motions of the 30 head $^{10,28}$ and asked how quickly their intermediate translocation 
Figure 2 Effect of the tRNAs on the large-angle $30 \mathrm{~S}$ body rotations. (a) Schematic of the tRNAL5-S13 interaction network. (b) Histogram of intersubunit interaction enthalpy for bridge $B 1 b$ residues, calculated from simulations of the pre5b structural model with (blue) and without tRNA (green). (c) Histogram of respective rotation angles derived from MD simulations. The arrow marks the angle of the starting structure as reference. (d) Histogram of $30 \mathrm{~S}$ body-rotation angles derived from cryoEM analysis of ribosomes in the absence of tRNAs (green) compared to those of states pre1 to pre5 (cyan).

states interconvert. To this end, we determined pivot points, rotation axes and angles for $30 \mathrm{~S}$ head and body motion (Fig. 1e; reaction coordinates described in Supplementary Note 2). During the transition from pre1 to pre5, the angles of $30 \mathrm{~S}$ head tilting and body rotation gradually increase before returning to low values in the pre5post 1 transition. In the post 3 state, the rotation angles are even lower than in pre 1 or post 1 , and they finally return to their initial value in post 4 , thus closing the conformational cycle of the 30 S subunit due to tRNA release from the $\mathrm{E}$ site. As can be seen from the amplitude of rapid $\left(>10^{7} / \mathrm{s}\right)$ fluctuations extracted from the simulations, large-scale motions occur not only on the millisecond time scale accessible to single-molecule experiments ${ }^{29}$ but also on the submicrosecond time scale. Moreover, the large overlaps between the fluctuation amplitudes of several adjacent states suggest fast transitions between these states, in particular for $30 \mathrm{~S}$ head swiveling. Conversely, lack of an overlap between adjacent states (for example, between pre 4 and pre 5 or between pre 5 and post 1 for $\mathrm{tRNA}^{\mathrm{Val}}$ ) indicates transitions between states that are slower than $100 \mathrm{~ns}$.

\section{Slow tRNA movement governs overall transition rates}

To determine which of the observed motions limit the overall interconversion rates between the states, we quantified the overlaps between all states and translated them into free-energy barriers and intrinsic (order of magnitude) transition times of the individual collective motions (Fig. 1d, Supplementary Note 2 and Supplementary Fig. 3). Whereas individual motions may be intrinsically fast in isolation, their coupling to slower motions limits the respective overall transition rates. Accordingly, we used the slowest transition between each pair of states in Figure 1d to estimate the overall transition rates. For all transitions between states with the exception of post1-post2, the slowest estimated rate is markedly slower than microseconds. This result agrees with rates determined by bulk kinetics ${ }^{30,31}$ and single-molecule studies $^{32-34}$ for tRNA translocation (milliseconds) as well as with those for L1 stalk-tRNA interactions ${ }^{12}$ and intersubunit rotation ${ }^{29}$ (seconds). A cluster of high free-energy barriers (i.e., slow transition times) is found at the transition from pre to post states, thus supporting the notion that the pre-to-post transition is the rate-limiting step of spontaneous translocation ${ }^{5,10}$. For the tRNA transitions, low transition barriers for tRNA ${ }^{\mathrm{Val}}$ within the pre and post states mostly correlate with high barriers for tRNA ${ }^{\text {fMet }}$ and vice versa. Together with the fact that the movements of the two tRNAs are coupled by base-pairing to the mRNA (Supplementary Fig. 4), this finding implies that, although intrinsically rapid, the excursions of tRNA ${ }^{\text {fMet }}$ between adjacent states (for example, pre5b and post2a) are governed by the slow movement of tRNA ${ }^{\text {Val }}$. Therefore, tRNA motion governs not only the overall rate of translocation ${ }^{35}$ but also, to a large extent, the dynamics within the pre and post states. In contrast, intrinsic rates for L1-stalk dynamics were present in the submicrosecond range, except for the slower movement from pre to post and from pre2 to pre3. Notably, our simulations revealed a large number of alternative microsecond and submicrosecond transitions of the tRNAs and the L1 stalk (Fig. 1d).

\section{Rapid intersubunit rotations couple to slow tRNA movement}

All individual transition barriers for $30 \mathrm{~S}$ motions turned out to be remarkably low, such that the intrinsic motions of the 30 S subunit, despite its large size, take place in the submicrosecond range, similarly to the movements of the L1 stalk. The slowest $30 \mathrm{~S}$ head transitions were found between states pre 2 and pre 3 as well as between pre 5 and post1, consistent with barriers inferred from cryo-EM and biochemical experiments ${ }^{5,10}$. The abundance of low barriers for motions of individual components seems to be a general feature of ribosome dynamics, and this underscores the important role of coupling between collective motions of both tRNAs and the ribosome ${ }^{3}$.

One example of such coupling is the stabilization of high intersubunit rotation by tRNAs, which has been previously observed ${ }^{29}$. Our simulations showed that, in state pre5b, the large head tilting and body rotation (angles at $\sim 20^{\circ}$ and $\sim 15^{\circ}$, Fig. 1e) are stabilized by strong interactions involving the tRNAs and a network of contacts between the two subunits, comprising L 5 on the 50 S subunit and S13 and S19 on the 30 S subunit (Figs. $\mathbf{1 c}$ and $\mathbf{2 b}$ ). In the classical state, these interactions form bridge $\mathrm{B}^{1} \mathrm{~b}^{13}$ (sketched in Fig. 2a). Removal of the tRNAs in state pre $5 \mathrm{~b}$ should thus weaken these intersubunit interactions with respect to the prel state. As a consequence, the highly rotated states should also be destabilized, and a smaller population of large rotation angles should thus be observed. To test this hypothesis, we carried out additional MD simulations of ribosomes in the pre5b state from which the tRNAs were removed (Fig. 2b,c; rapid angular rearrangement described in Supplementary Note 3). These simulations indeed showed weakened interactions between the B1b residues (Fig. 2a,b) as well as decreased $30 \mathrm{~S}$ body-rotation angles (Fig. 2c and Supplementary Fig. 5). Indeed, additional cryo-EM data of vacant ribosomes (Supplementary Note 1) revealed a markedly reduced population of high bodyrotation angles ( $\sim 50 \%$ for angles of $10^{\circ}$ and above, Fig. $\left.2 \mathbf{d}\right)$. Apparently, our structural models and simulations are sufficiently accurate to capture the underlying small energy differences. A similar effect 


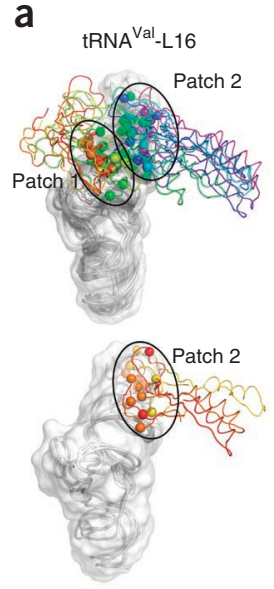

tRNA $^{\mathrm{fMet}}-\mathrm{L} 16$

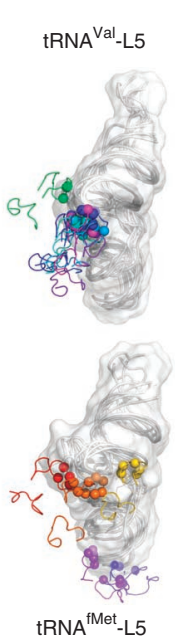

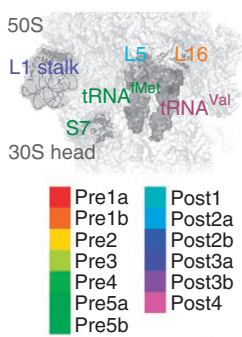

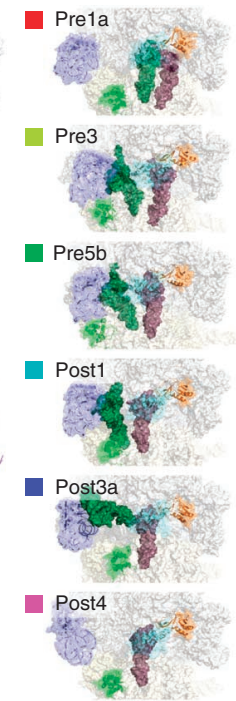

b
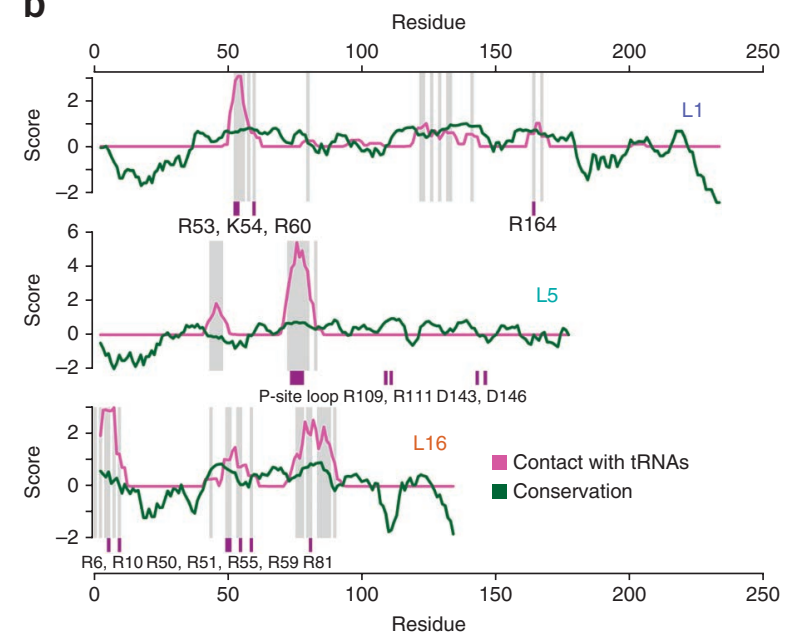

Figure 3 Handover of tRNAs by 50S-subunit proteins during translocation. (a) Left, positions and key interacting residues (spheres) of the three main interaction partners, L16, L5 and L1 (ribbons, colored according to the state), relative to tRNAVal (top) and tRNA fMet (bottom). Right, selected conformations relative to the $50 \mathrm{~S}$ subunit, including the position of S7 on the $30 \mathrm{~S}$ head for reference. (b) Conservation of protein residues contacting tRNAs. Conservation score (higher, more conserved) and contacts score (higher, more frequent contacts) for the L1, L5 and L16 proteins. The scores were smoothed with a 5-aa sliding window. Residues considered contacting are marked with a gray background. Residues highlighted in purple are those specifically mentioned in the text.

on body rotation should be seen for weakened intersubunit interactions, for example, in mutation of the charged L5 residues R109, R111, D143 and D146 to uncharged residues. Indeed, mutation of the corresponding residues in yeast affects translational fidelity ${ }^{36}$. Taken together, these results indicate that previously observed correlated motions ${ }^{29}$ are causally connected and explain this coupling in terms of molecular interactions.

\section{Kinetic sequence of translocation intermediate states}

The transition-rate estimates determined from the simulations (Fig. 1d and Supplementary Fig. 6a) enabled us to add time information to the sequence of states that was previously determined by Fischer et al. ${ }^{10}$ from structural similarity only. To single out the sequence of states that best reflects the motion of the ribosome along the translocation pathway from all $(13-1) !=479,001,600$ possible linear kinetic sequences that can be formed from permutations of the 12 available conformational states with two tRNAs, we calculated for each of these sequences the overall progression rate from the barrier heights for all five ribosomal components shown in Figure 1a. The sequences with the fastest progression rates turned out to be very similar to the one obtained purely from structural similarity of the tRNAs, thus showing that this sequence reflects the kinetics of the system (kinetic sequence of states in Supplementary Note 2). Interestingly, removal of any state from this sequence (except pre5b) slows down the progression rate, a result underscoring the kinetic relevance of all states (with the possible exception of pre5b). A systematic scan through all 31 possible subsets of the five conformational motions considered in Figure 1 (Supplementary Note 2 and Supplementary Fig. 6b) confirms the initial suggestion (based on Fig. 1d) that translocation is limited by the motion of the tRNAs. We note that although the preferential overall direction of movement in our experiment is backwards, Fischer et al. ${ }^{10}$ found that different substates within the ensembles of pre or post states were in rapid equilibrium, and thus at any given time the ribosomes were undergoing transitions in both directions, such that forward and backward directions were equivalent. Similarly, detailed balance also holds for our simulations.

\section{L1 stalk links 30S rotation to translocation by 'pulling' tRNA}

Having identified the tRNA motions as the main determinant for translocation efficiency, we expected to find mechanisms that accelerate the rates of these translocation motions. One obvious candidate involves the L1 stalk, which has been suggested to be important for translocation ${ }^{3,4}$. The L1 stalk forms contacts to the tRNA ${ }^{4,11-13,15,16}$ as well as to proteins S7 (30S head) and S11 (30S body) (Figs. 1c, 3 and 4). In the pre 1 and pre 2 states, the L1 stalk is found in an open conformation. In the pre3 state, interactions with $S 11$ are weakened, and the $30 \mathrm{~S}$ head tilts (Fig. 1e), moving S7 into a position that allows it to contact the L1 stalk in the closed conformation. This presumably shifts the L1-stalk equilibrium toward the E site, where it forms strong contacts to tRNA ${ }^{\text {fMet }}$ (Fig. 1c). Upon decreasing of the tilting angle in post1, contact to S7 is lost, and the L1 stalk moves toward the open

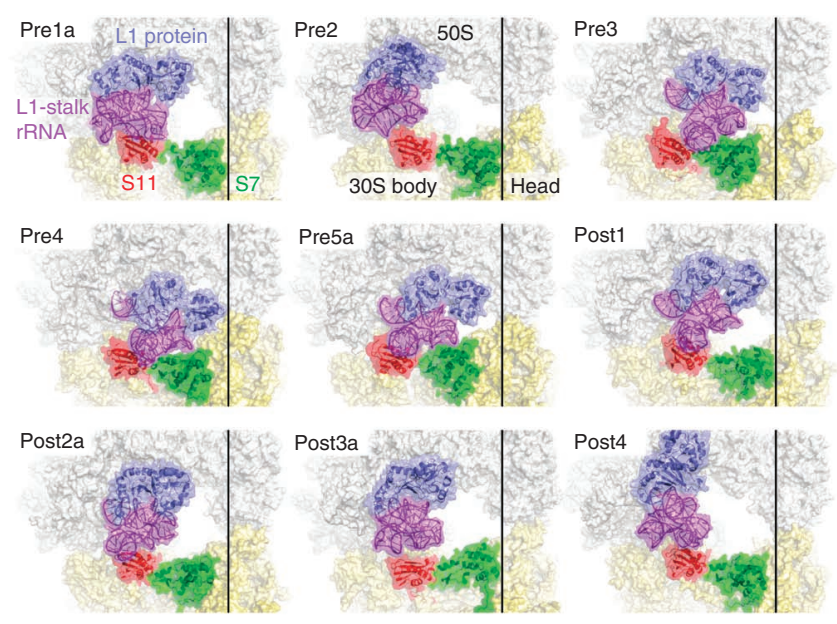

Figure 4 L1-stalk contacts with 30S-subunit proteins S7 and S11. For each state, protein L1 (blue) and L1-stalk rRNA (magenta) as well as proteins S7 (green) and S11 (red) are depicted as ribbons. The 50S and 30 s subunits are shown as gray and yellow surfaces. For clarity, the tRNAs are not shown. The vertical black lines serve as reference. 
conformation, maintaining its contact to the tRNA ${ }^{\text {fMet }}$ throughout the post states.

Because structural analyses alone do not provide evidence on whether the L1 stalk accelerates tRNA translocation, we needed to establish that the concerted motion is actually driven by the L1 stalk and not by the tRNA. We calculated the underlying molecular driving forces from our equilibrium simulations of each state (interaction enthalpy) as well as from additional umbrella-sampling simulations (L1 stalk-tRNA ${ }^{\text {fMet }}$ potential of mean force, Supplementary Note 2). The interaction enthalpy and binding free energy changed with the distance between the L1 stalk and tRNA ${ }^{\text {fMet }}$ (Fig. 1f). The positive slopes imply that both the interaction enthalpy and free energy are attractive. These two independent data sets suggest that the L1 stalk actively pulls the tRNA after contact is established in the pre3 state, thereby accelerating tRNA barrier crossing. The opposite repulsive scenario, i.e., the tRNA pushing the L1 stalk, which would slow down barrier crossing, is incompatible with the observed free-energy curve. This result explains both the lower translation rates ${ }^{37}$ and the higher stability of the classical tRNA states ${ }^{14}$ observed for L1-depleted ribosomes, and it links 30S body and head rotation through the L1 stalk to the motion of the tRNA. That the total interaction enthalpy (Fig. 1f) shows the same distance dependency as the binding free energy (albeit with larger values due to partial enthalpy-entropy compensation) suggests that the analysis of interaction enthalpies, to a good approximation, can be used to reveal main interaction sites.

A closer analysis of the interaction enthalpies seen between L1 and the tRNA suggests residues R53, K54, R60 and R164 of L1, which are highly conserved (Fig. 3b), as the main interaction sites. L1 residues R53, R60 and R164 contacted residue $\Psi 55$ of the tRNA $^{\text {fMet }}$ (Supplementary Table 3). Mutations of the $\Psi 55 \cdot G 18$ base pair are known to decrease translocation rates by 80 -fold ${ }^{38}$, thus supporting the notion that attractive interactions with the L1 stalk actively decrease the barrier for tRNA translocation. A similar effect would be expected from a complementary mutation of the identified $\mathrm{L} 1$ residues.

\section{L5 and L16 facilitate tRNA translocation}

During translocation, the two tRNAs are handed over from protein L16 to L5 and then to L1. We identified the strong tRNA interactions (identified by enthalpy contributions, Fig. 1c) with these largesubunit proteins and analyzed how the position, structure and contact sites of these proteins change from the perspective of the tRNAs in each state along the tRNA pathway from the A to the E site (Fig. 3a). Notably, more state-specific contacts to the tRNAs were generally seen for ribosomal proteins than for rRNA (Supplementary Note 3). Considerable motions of all involved components are observed; nevertheless, the tRNA remains tightly bound to these proteins, thus enabling accurate adjustment of the tRNA binding free energy. The continuous sequence of interactions might serve to lower free-energy barriers that otherwise would impede tRNA translocation. Indeed, sequence analysis of proteins L1, L5 and L16 revealed significantly higher conservation of the residues identified by contact analysis (overall $P$ value $=6.62 \times 10^{-8}$ obtained from a one-sided permutation test in which the sample comprised $n=550$ individual amino acids from the proteins), thus corroborating their functional relevance (Fig. 3b; conservation of contact residues described in Supplementary Note 2).

How are the large structural motions reconciled with the maintained interactions between the tRNA and the ribosome along the translocation path? Closer inspection of interactions revealed two main mechanisms: stepping and sliding. During the A/A-to-P/P
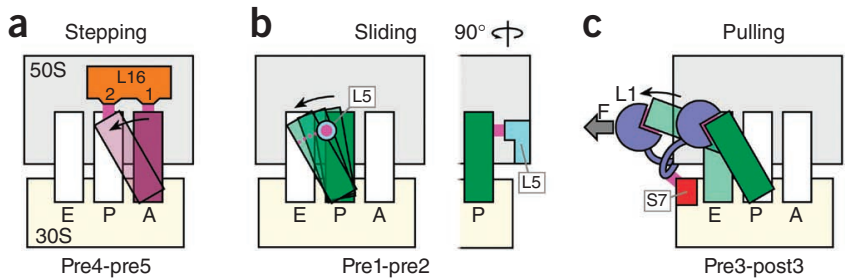

Figure 5 Three mechanisms facilitating tRNA translocation. (a) Two distinct contact patches of L16 stabilize A- and P-site positioning of the tRNA (here tRNAVal, purple) on the 50S subunit. Moving from A to $P$ site, the tRNA steps from patch 1 to patch 2. (b) As the tRNA moves

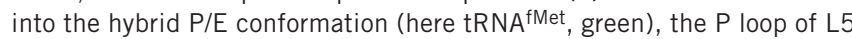
maintains a flexible contact (magenta) by sliding along the tRNA (green). (c) In pre3, high intersubunit rotations stabilize the closed L1-stalk conformation through S7. After back rotation (post1), this interaction is lost, and the L1 stalk moves into the open conformation, thereby pulling the tRNA into the $E$ site.

transition, tRNA ${ }^{\mathrm{Val}}$ remained in contact with L16 (Figs. 3a and 5). We used two adjacent interaction patches on L16 (R50, R51, R55 and R59; R6, R10 and R81), each involving positively charged, highly conserved arginines (Fig. $3 \mathbf{b}$ ) that interacted with different parts of the negatively charged tRNA backbone. Upon hybrid-state formation (pre4 to pre5)-when the acceptor stem of tRNAVal moved into the $\mathrm{P}$ site of the $50 \mathrm{~S}$ subunit while the L16 conformation remained unchanged-the contact region on $\mathrm{tRNA}^{\mathrm{Val}}$ switched from the first to the second patch. We observed similar interactions with the second patch on L16 for the tRNA ${ }^{\mathrm{fMet}}$ in its $\mathrm{P} / \mathrm{P}$ configuration, suggesting that these contacts can stabilize any tRNA in the P site. During the tRNA handover from L16 to L5, the binding of tRNA ${ }^{\text {Val }}$ to L16 became weaker (Fig. 1c), whereas the binding to L5 remained strong in pre5b and throughout the post states. Strong contacts between the tRNAVal C56 and the highly conserved P-site loop of L5 (ref. 39) (A74-I78, Fig. 3b) were also present in the pre5b state. This finding, together with the large intersubunit rotation angle in the pre5b state, suggests that the tRNA movement is coupled to the intersubunit rotation through L5. In the subsequent post states, the contacts of the L5 P-site loop shifted down the D loop of the tRNA ${ }^{\mathrm{Val}}$ (Fig. 3a), additionally involving nucleotide G19.

In contrast to interactions involved in the stepping motion of the rather rigid protein L16, interactions between $\mathrm{tRNA}^{\mathrm{fMet}}$ and L5 (Fig. 3a) were much less localized and were more dynamic. In the pre 1 and pre 2 states, tRNA $^{\text {fMet }}$-L5 contacts shifted from the D loop to the T loop of the tRNA. Upon handover to the L1 stalk in the pre3 state, these contacts to L5 were lost, but new contacts between L5 and the anticodon stem-loop were formed just before the tRNA left the ribosome (in post4). Here, the P-site loop of L5 slides smoothly over the tRNA, thereby flexibly adapting to the changing tRNA position and orientation. Mutations of the P-site loop of L5 in yeast ribosomes impair tRNA binding ${ }^{39}$, underscoring the importance of L5 as a guide for the movement of the P-site tRNA. The L1 stalk provides the final contact for tRNA ${ }^{\text {fMet }}$ after being handed over from L5 and L16. Interactions of L1 with tRNA ${ }^{\mathrm{fMet}}$, involving the tRNA backbone and the highly conserved, positively charged $\mathrm{L} 1$ residues (R53, $\mathrm{K} 54, \mathrm{R} 60$ and R164), were established in the pre3 state and remained unchanged from pre3 to post 2 .

\section{DISCUSSION}

Each of the three different mechanisms facilitating tRNA translocation (summarized in Fig. 5) rests on mutual couplings between the tRNAs and parts of their binding region. First, the precise positioning 
of the tRNAs in A and P sites is achieved by interactions with L16. To accommodate tRNA motions, L16 'steps' through discontinuous contact patches. Second, the flexible P-site loop of L5 facilitates seamless tRNA sliding across the P site. Third, L1 exerts force to pull the tRNA out of the $\mathrm{P}$ site and hence requires internal rigidity. From real-time single-molecule fluorescence resonance energy transfer experiments, the L1 stalk is known to remain associated with the tRNA throughout translocation ${ }^{12}$. Our analysis suggests that L1 forms only one stable, highly conserved contact patch with the tRNA and moves with it as a rather rigid body. The L1 stalk transmits the intersubunit rotation through S7-L1 stalk interactions and promotes tRNA translocation by reducing free-energy barriers between adjacent binding sites. The abundance of interactions between charged, highly conserved residues of ribosomal proteins and the tRNA backbone may provide a general means for the transport of different tRNA species, independently of their sequence, through the ribosome.

Although translocation is a function inherent to the ribosome itself $^{7,8}$, the movement, particularly on the $30 \mathrm{~S}$ subunit, is dramatically facilitated by EF-G. Although the full description of EF-G-dependent translocation is currently not feasible, owing to the lack of structural information, the present work provides insights into the reaction landscape underlying the movement. The simulations provide a view of the energy 'valley' of spontaneous translocation, which allows the tRNAs to move by large distances while maintaining sufficient binding with the ribosome components. Gross deviations from this overall pathway would require a very large energy input and thus seem unlikely even in the presence of EF-G. Rather, EF-G might use the energy of binding and GTP hydrolysis to flatten the energy barriers of the rate-limiting step(s) and to provide the directional bias for forward movement. Understanding the way by which EF-G remodels the free-energy landscape into efficient tRNA translocation is one of the most important future challenges in the field.

Our combined crystallography-cryo-EM-simulation approach reveals fast, large-scale motions of the ribosome, on microsecond time scales, that govern tRNA translocation. Where the small overlap between the fast time scales accessible here and the slower ones observable by bulk kinetics ${ }^{30,31}$ and single-molecule studies ${ }^{12,29,32-34}$ allows a direct comparison, the obtained transition rates agree within the respective error bounds. Our approach quantifies, from first principles, the picture of a stochastic molecular machine ${ }^{10,30,33}$, which fluctuates ${ }^{12,14,15,29,35}$ between nearly isoenergetic Frauenfelder-type conformational states through collective and coupled structural transitions ${ }^{40}$ (Supplementary Video 3 ). Of all the movements described above, the highest intrinsic barriers are found for tRNA movement throughout the whole translocation pathway. This finding suggests a possible explanation for the strong effect of different tRNA species ${ }^{5,6}$ on the rate of spontaneous translocation. Our picture shows how the 30 S head and body rotations, through coordinated L1-stalk, S7 and S11 motions, promote and control tRNA translocation. This complements structural $1,3,4,10,11,13,18,26,28,36,39,41-43$ and single-molecule data ${ }^{12,14,15}$ with intrinsic transition rates of functionally relevant and coupled motions, interaction energies and the underlying molecular driving forces. We begin to uncover the 'gears and wheels' of tRNA translocation through the ribosome from first principles, in terms of a stochastic molecular machine.

\section{METHODS}

Methods and any associated references are available in the online version of the paper.
Accession codes. Three-dimensional density maps have been deposited at the Electron Microscopy Data Bank, under accession codes EMD-2472 (pre1b), EMD-2473 (pre5a), EMD-2474 (post2b), EMD-2475 (post3a). The atomic coordinates have been deposited at the Protein Data Bank under accession codes 3J4V, 3J52 (prela); 3J4W, 3J4X (pre1b); 3J4Z, 3J50 (pre2); 3J4Y, 3J51 (pre3); 3J53, 3J54 (pre4); 3J55, 3J56 (pre5a); 3J57, 3J58 (pre5b); 3J59, 3J5A (post1); 3J5B, 3J5C (post2a); 3J5D, 3J5E (post2b); 3J5F, 3J5G (post3a); 3J5H, 3J5I (post3b); and 3J5J, 3J5K (post4). Details can be found in Supplementary Table 4.

Note: Any Supplementary Information and Source Data files are available in the online version of the paper.

\section{ACKNOWLEDGMENTS}

We thank B. de Groot, U. Zachariae, C. Kutzner, R. Jahn, G. Hummer, B. Roux, W. Wintermeyer and C. Rotte for discussions and critical reading of the manuscript. M.V.R. and H.S. acknowledge financial support of the Deutsche Forschungsgemeinschaft (DFG) (Sonderforschungsbereich grant 860 and DFG-Forschergruppe 1805). Financial support for L.V.B., C.B., A.C.V. and H.G. comes from the Max Planck Society, International Max Planck Research School for Physics of Biological and Complex Systems and DFG-Forschergruppe 1805. We thank the computer center Garching (RZG) and the Gesellschaft fuer wissenschaftliche Datenverarbeitung Goettingen (GWDG) for technical assistance; computer time has been provided by the RZG.

\section{AUTHOR CONTRIBUTIONS}

L.V.B. and A.C.V. prepared the ribosome model; G.F.S. refined the model against the cryo-EM maps; L.V.B. performed the molecular dynamics simulations; L.V.B. and C.B. analyzed the data with mentoring by A.C.V. and H.G.; C.B., L.V.B., A.C.V. and H.G. developed and implemented analysis methods for rate estimation; I.I.D. carried out the sequence-conservation analysis; N.F. performed the cryoEM experiments and analysis; H.S., H.G. and M.V.R. conceived of the project. All authors discussed the results and wrote the manuscript.

\section{COMPETING FINANCIAL INTERESTS}

The authors declare no competing financial interests.

Reprints and permissions information is available online at http://www.nature.com/ reprints/index.html.

1. Moazed, D. \& Noller, H.F. Intermediate states in the movement of transfer RNA in the ribosome. Nature 342, 142-148 (1989).

2. Frank, J. \& Agrawal, R.K. A ratchet-like inter-subunit reorganization of the ribosome during translocation. Nature 406, 318-322 (2000).

3. Tama, F., Valle, M., Frank, J. \& Brooks, C.L. Dynamic reorganization of the functionally active ribosome explored by normal mode analysis and cryo-electron microscopy. Proc. Natl. Acad. Sci. USA 100, 9319-9323 (2003).

4. Valle, M. et al. Locking and unlocking of ribosomal motions. Cell 114, 123-134 (2003).

5. Konevega, A.L. et al. Spontaneous reverse movement of mRNA-bound tRNA through the ribosome. Nat. Struct. Mol. Biol. 14, 318-324 (2007).

6. Shoji, S., Walker, S.E. \& Fredrick, K. Reverse translocation of tRNA in the ribosome. Mol. Cell 24, 931-942 (2006).

7. Spirin, A.S. Energetics of the ribosome. Prog. Nucleic Acid Res. Mol. Biol. 21 39-62 (1978).

8. Noller, H.F., Yusupov, M.M., Yusupova, G.Z., Baucom, A. \& Cate, J.H. Translocation of tRNA during protein synthesis. FEBS Lett. 514, 11-16 (2002).

9. Liu, Q. \& Fredrick, K. Contribution of intersubunit bridges to the energy barrier of ribosomal translocation. Nucleic Acids Res. 41, 565-574 (2013).

10. Fischer, N., Konevega, A.L., Wintermeyer, W., Rodnina, M.V. \& Stark, H. Ribosome dynamics and tRNA movement by time-resolved electron cryomicroscopy. Nature 466, 329-333 (2010).

11. Agirrezabala, X. et al. Visualization of the hybrid state of tRNA binding promoted by spontaneous ratcheting of the ribosome. Mol. Cell 32, 190-197 (2008).

12. Fei, J., Kosuri, P., MacDougall, D. \& Gonzalez, R. Coupling of ribosomal L1 stalk and tRNA dynamics during translation elongation. Mol. Cell 30, 348-359 (2008).

13. Julián, P. et al. Structure of ratcheted ribosomes with tRNAs in hybrid states. Proc. Natl. Acad. Sci. USA 105, 16924-16927 (2008).

14. Munro, J.B., Altman, R.B., O'Connor, N. \& Blanchard, S.C. Identification of two distinct hybrid state intermediates on the ribosome. Mol. Cell 25, 505-517 (2007). 
15. Cornish, P.V. et al. Following movement of the L1 stalk between three functional states in single ribosomes. Proc. Natl. Acad. Sci. USA 106, 2571-2576 (2009)

16. Trabuco, L.G. et al. The role of L1 stalk-tRNA interaction in the ribosome elongation cycle. J. Mol. Biol. 402, 741-760 (2010).

17. Sanbonmatsu, K.Y., Joseph, S. \& Tung, C.-S. Simulating movement of tRNA into the ribosome during decoding. Proc. Natl. Acad. Sci. USA 102, 15854-15859 (2005).

18. Zhang, W., Dunkle, J.A. \& Cate, J.H.D. Structures of the ribosome in intermediate states of ratcheting. Science 325, 1014-1017 (2009).

19. Caulfield, T.R., Devkota, B. \& Rollins, G.C. Examinations of tRNA range of motion using simulations of cryo-EM microscopy and X-ray data. J. Biophys. 2011, 219515 (2011).

20. Tama, F., Miyashita, O. \& Brooks, C.L. Flexible multi-scale fitting of atomic structures into low-resolution electron density maps with elastic network normal mode analysis. J. Mol. Biol. 337, 985-999 (2004).

21. Whitford, P.C. et al. An all-atom structure-based potential for proteins: bridging minimal models with all-atom empirical forcefields. Proteins 75, 430-441 (2009).

22. Tan, R.K.Z., Devkota, B. \& Harvey, S.C. YUP.SCX: coaxing atomic models into medium resolution electron density maps. J. Struct. Biol. 163, 163-174 (2008).

23. Schröder, G.F., Brunger, A. \& Levitt, M. Combining efficient conformational sampling with a deformable elastic network model facilitates structure refinement at low resolution. Structure 15, 1630-1641 (2007).

24. Ahmed, A. \& Tama, F. Consensus among multiple approaches as a reliability measure for flexible fitting into cryo-EM data. J. Struct. Biol. 182, 67-77 (2013).

25. Orzechowski, M. \& Tama, F. Flexible fitting of high-resolution X-ray structures into cryoelectron microscopy maps using biased molecular dynamics simulations. Biophys. J. 95, 5692-5705 (2008)

26. Dunkle, J.A. et al. Structures of the bacterial ribosome in classical and hybrid states of tRNA binding. Science 332, 981-984 (2011).

27. de Groot, B.L., Engel, A. \& Grubmuller, H. The structure of the aquaporin-1 water channel: a comparison between cryo-electron microscopy and X-ray crystallography. J. Mol. Biol. 325, 485-493 (2003).

28. Schuwirth, B.S. et al. Structures of the bacterial ribosome at $3.5 \mathrm{~A}$ resolution. Science 310, 827-834 (2005).
29. Cornish, P.V., Ermolenko, D.N., Noller, H.F. \& Ha, T. Spontaneous intersubunit rotation in single ribosomes. Mol. Cell 30, 578-588 (2008).

30. Rodnina, M.V., Savelsbergh, A., Katunin, V.I. \& Wintermeyer, W. Hydrolysis of GTP by elongation factor $\mathrm{G}$ drives tRNA movement on the ribosome. Nature 385, 37-41 (1997).

31. Pan, D., Kirillov, S.V. \& Cooperman, B.S. Kinetically competent intermediates in the translocation step of protein synthesis. Mol. Cell 25, 519-529 (2007).

32. Fei, J., Richard, A.C., Bronson, J.E. \& Gonzalez, R.L. Transfer RNA-mediated regulation of ribosome dynamics during protein synthesis. Nat. Struct. Mol. Biol. 18, 1043-1051 (2011)

33. Munro, J.B., Sanbonmatsu, K.Y., Spahn, C.M. \& Blanchard, S.C. Navigating the ribosome's metastable energy landscape. Trends Biochem. Sci. 34, 390-400 (2009).

34. Chen, C. et al. Single-molecule fluorescence measurements of ribosomal translocation dynamics. Mol. Cell 42, 367-377 (2011).

35. Blanchard, S.C., Kim, H.D., Gonzalez, R.L. Jr., Puglisi, J.D. \& Chu, S. tRNA dynamics on the ribosome during translation. Proc. Natl. Acad. Sci. USA 101 12893-12898 (2004).

36. Rhodin, M.H.J. \& Dinman, J.D. An extensive network of information flow through the $\mathrm{B} 1 \mathrm{~b} / \mathrm{c}$ intersubunit bridge of the yeast ribosome. PLOS ONE 6, e20048 (2011).

37. Subramanian, A.R. \& Dabbs, E.R. Functional studies on ribosomes lacking protein L1 from mutant Escherichia coli. Eur. J. Biochem. 112, 425-430 (1980).

38. Pan, D., Kirillov, S., Zhang, C.M., Hou, Y.M. \& Cooperman, B.S. Rapid ribosomal translocation depends on the conserved $18-55$ base pair in P-site transfer RNA. Nat. Struct. Mol. Biol. 13, 354-359 (2006).

39. Rhodin, M.H.J. \& Dinman, J.D. A flexible loop in yeast ribosomal protein L11 coordinates P-site tRNA binding. Nucleic Acids Res. 38, 8377-8389 (2010).

40. Frauenfelder, H., Sligar, S.G. \& Wolynes, P.G. The energy landscapes and motions of proteins. Science 254, 1598-1603 (1991).

41. Yusupov, M.M. et al. Crystal structure of the ribosome at $5.5 \AA$ resolution. Science 292, 883-896 (2001).

42. Gao, Y.G. et al. The structure of the ribosome with elongation factor $F$ trapped in the posttranslocational state. Science 326, 694-699 (2009).

43. Budkevich, T. et al. Structure and dynamics of the mammalian ribosomal pretranslocation complex. Mol. Cell 44, 214-224 (2011). 


\section{ONLINE METHODS}

The ribosome model was based on a crystal structure by Zhang et al. (PDB 3I1P and $3 \mathrm{IIO})^{18}$; the crystal structure by Gao et al. (PDB 2WRI) ${ }^{42}$ was used to model the L1 stalk. To match the cryo-EM setup ${ }^{10}$, tRNA structures ${ }^{42,44}$ were incorporated into the model. Models were then refined against 13 cryo-EM maps ${ }^{10}$ with the real-space refinement program DireX ${ }^{23}$. Explicit-solvent all-atom molecular dynamics simulations were carried out with GROMACS ${ }^{45}$ using the amber99sb force field ${ }^{46}$. R.m.s. deviations of refined models relative to crystal structures of the ground-state and rotated structure ${ }^{26}$ were calculated after rigid-body fitting, using (i) all $70 \mathrm{~S} \mathrm{C} \alpha$ and $\mathrm{P}$ atoms, except L9 protein atoms, and (ii) all atoms within a 2-nm distance from the two tRNAs. To obtain reaction coordinates for the tRNAs and the L1 stalk, principal component analysis (PCA) was performed on the trajectories. Axes and rotation angles of $30 \mathrm{~S}$ head and body rotations were obtained from each frame of the trajectories by nonlinear least-squares fitting and then averaged. Initial free energy-barrier heights between different states were estimated with multidimensional transition-state theory, on the basis of atomic fluctuations obtained from the simulations. All barrier heights and transitionrate prefactors were calibrated with barrier crossings that were fast enough to be observed in the simulations. Interaction enthalpies were obtained directly from the force field. The potential of mean force between the L1 stalk and the tRNA was obtained by umbrella-sampling simulations.

Vacant E. coli ribosomes were prepared for cryo-EM at $18^{\circ} \mathrm{C}$ and imaged with a Titan Krios electron microscope (FEI Company) on a 4,000 × 4,000 CCD camera (FEI company) using two-fold pixel binning ( $3.2 \AA$ A per pixel). The resulting 9,814 ribosome particles and 315,108 pretranslocation-state E. coli ribosome particles from an existing cryo-EM data set ${ }^{10}$ were analyzed and classified according to $30 \mathrm{~S}$ body rotation as described. A detailed description of experimental and computational methods is provided in Supplementary Note 1.

44. Grishaev, A., Ying, J., Canny, M., Pardi, A. \& Bax, A. Solution structure of tRNA Val from refinement of homology model against residual dipolar coupling and SAXS data. J. Biomol. NMR 42, 99-109 (2008).

45. Hess, B., Kutzner, C., van der Spoel, D. \& Lindahl, E. GROMACS 4: algorithms for highly efficient, load-balanced, and scalable molecular simulation. J. Chem Theory Comput. 4, 435-447 (2008).

46. Hornak, V. et al. Comparison of multiple Amber force fields and development of improved protein backbone parameters. Proteins 65, 712-725 (2006). 